\title{
Bei Diabetikern ist die Schilddrüse besonders im Fokus
}

\begin{abstract}
Bei Menschen mit Diabetes kann eine Schilddrüsenüberfunktion die Insulinsekretion weiter verschlechtern. Eine Hypothyreose wiederum erhöht das Risiko für Unterzuckerungen. Umgekehrt beeinflusst ein schlecht eingestellter Diabetes auch die Schilddrüsenfunktion negativ. Hier ist vermehrte Aufmerksamkeit gefragt.
\end{abstract}

Wie wirken sich Schilddrüsenfunktionsstörungen auf den Glukosestoffwechsel bei Menschen mit Diabetes mellitus aus? Eine Hypothyreose führt bei Diabetikern zu einer gesteigerten Insulinsensitivität. Der tägliche Insulinbedarf der Patienten sinkt, erläuterte Dr. Alexandra Willms, Internistin am Klinikum München Bogenhausen. Es komme zu einer herabgesetzten gastrointestinalen Motilität und Glukoseaufnahme und man habe eine verstärkte Hypoglykämieneigung beobachtet.

Anhand von Studiendaten demonstrierte Wilms, wie sich eine subklinische Hypothyreose auf die Stoffwechselkontrolle bei Kindern und Jugendlichen mit Typ-1-Diabetes $(n=13)$ im Vergleich zur Kontrollgruppe (Typ-1-Diabetes und Euthyreose: $n$ = 31) auswirkte: Während $\mathrm{HbA}_{1 \mathrm{c}}$-Werte und Insulinbedarf sich in beiden Gruppen nicht signifikant unterschieden, kam es bei Hypothyreose zu einer deutlich höheren Hypoglykämierate, die unter Levothyroxin-Gabe rückläufig war ( $\triangle A b b .1)$ [1].

\section{Autoimmunthyreoiditis bei Typ-1-Diabetikern}

Pro Jahr entwickeln etwa $2-4 \%$ der Patienten mit positiven TPO-(thyreoidale-Peroxidase-)/Tg-(Thyreoglobulin-)Antikörpern eine subklinische/manifeste Hypothyreose. Da die Autoimmunthyreoiditis bei Typ-1-Diabetikern häufig ist, rät Wilms bei diesen Patienten zur jährlichen Kontrollen der TSHWerte, der TPO- und Tg-Antikörper.

Man sollte bei Menschen mit Typ-1-Diabetes bei entsprechenden Beschwerden auch immer an ein polyglanduläres Autoimmun-Syndrom denken, vor allem bei Typ-1-Diabetikerinnen, die vor kurzem entbunden haben.

\section{Schilddrüsenfunktion bei Typ-2-Diabetes}

Wilms: „Bei Typ-2-Diabetikern sollten unklare Stoffwechselentgleisungen bzw. unklare Befindlichkeitsstörungen zu einer Abklärung der Schilddrüsenfunktion führen, denn eine Hyperthyreose oder auch Hypothyreose können dahinter stecken."

Zudem unterstrich die Referentin, dass bereits bei subklinischer Hypothyreose ein Viertel der Betroffenen über Beschwerden klagt, darunter trockene Haut, kognitive Funktionsstörungen, depressive Verstimmung, muskuläre Schwäche, Müdigkeit, Kälteintoleranz, Schwellungen im Gesicht, vor allem im Bereich der Augen, und Obstipation [2]. Eine Hyperthyreose wiederum kann eine Glukoseintoleranz bzw. Stoffwechselentgleisungen fördern. Sie geht einher mit verstärkter Insulinresistenz in der Peripherie, Hemmung der Insulinsekretion, vermehrter intestinaler Glukoseaufnahme, pathologischer Glukagonfreisetzung und verstärkter Glykogenolyse in der Leber, so Willms.

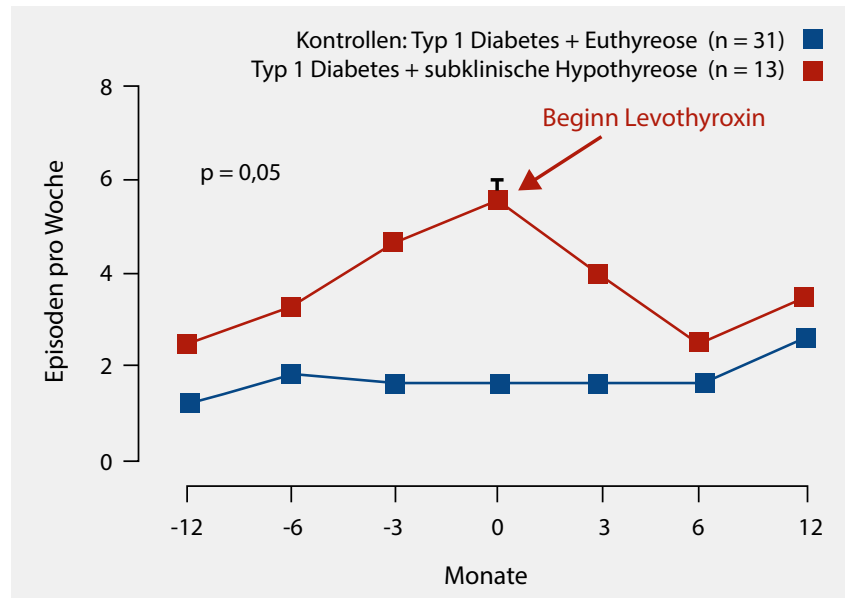

Hypoglykämierate/Monat (Maximal 5,5+0,4 vs 1,6+0,1 Episoden/Monat, $p=0,01$ )

1 Einfluss einer subklinischen Hypothyreose auf die Soffwechselkontrolle bei Kindern und Jugendlichen mit Diabetes Typ 1. Es kam zu einer deutlich höheren Hypoglykämierate, die unter LevothyroxinGabe rückläufig war.

Umgekehrt könne eine schlechte Glukosekontrolle bei Diabetikern auch die Schilddrüsenstoffwechsellage beeinflussen, sagte Wilms „Es kann bei schlecht eingestellten Diabetikern zu einem, Niedrig- $\mathrm{T}_{3}$-Syndrom ' kommen. “ Dieses ist gekennzeichnet durch niedriges $\mathrm{T}_{3}$ (Trijodthyronin)-Wert, erhöhtes $\mathrm{rT}_{3}$ (reverse $\mathrm{T}_{3}$ )-Wert bei normalem $\mathrm{T}_{4}$ (Thyroxin) und normalem basalem TSH [3[. „Das Niedrig-T ${ }_{3}$-Syndrom wird normalerweise nicht therapiert, da es eher als eine Art Schutzmechanismus des Körpers verstanden wird. “ Indiziert ist dagegen eine Stabilisierung der Glukosekontrolle.

Werner Zwick

Literatur:

1. Mohn et al. Diabetic Medicine 19 (2003): 70-3.

2. Canaris et al. Colorado Thyroid Disease Prevalence Study (2000).

3. Schumm-Draeger: Diabetes bei anderen endokrinen Erkrankungen. In: Diabetologie in Klinik und Praxis 2011.

Quelle: Symposium „Autoimmunerkrankungen und Diabetes mellitus" am 20.2.2016 in Unterschleißheim. „Innere Medizin fachübergreifend Diabetes grenzenlos" Veranstalter: Berufsverband Deutscher Internisten e.V. (BDI) und OmniaMed Deutschland GmbH. 